DOI: $\mathrm{xxx} / \mathrm{xxxx}$

\title{
ARTICLE TYPE \\ Resilience assessment of electrified road networks subject to charging station failures
}

\author{
Hongping Wang ${ }^{1}$ | Adam F. Abdin ${ }^{1}$ | Yi-Ping Fang*1 | Enrico Zio ${ }^{2,3,4}$
}

\footnotetext{
${ }^{1}$ Laboratoire Génie Industriel, Université Paris-Saclay, CentraleSupélec, 91190 Gif-sur-Yvette, France.

${ }^{2}$ Energy Department, Politecnico di Milano, 20156 Milano, Italy.

${ }^{3}$ Mines ParisTech, PSL Research

University,CRC, 06904 Sophia-Antipolis, France.

${ }^{4}$ Eminent Scholar, Department of Nuclear Engineering, Kyung Hee University, Republic of Korea.

*Correspondence

Yi-Ping Fang. Email:

yiping.fang@ centralesupelec.fr
}

\section{Present address}

Laboratoire Génie Industriel, Université Paris-Saclay, CentraleSupélec, 91190

Gif-sur-Yvette, France

\begin{abstract}
Summary
The number of EVs and charging facilities is expected to increase significantly in the near future, further coupling the existing transportation system with the power system. This may bring new stresses and risks to such system of systems. This paper presents a mathematical framework to analyze the resilience of an electrified road network (ERN) subject to potential failures of its supporting fast-charging stations (FCSs). Within this framework, a novel linear optimization model is proposed for the first time to solve the system optimal dynamic traffic assignment problem of ERN. The characteristics considered in the modeling framework include the location, capacity, and charging speed of FCSs, as well as the driving range, charging time and state of charge $(\mathrm{SoC})$ of EVs. The linear model is proposed based on the cell transmission model. It is used as the first stage model to assign the traffic under normal FCS operations. A second stage model is, then, extended to minimize the total travel time after the stochastic occurrence of FCS failures, i.e., in the failure and recovery phases. Two metrics are considered to quantify the ERN performance and the impacts of FCS failures. A numerical example is studied to illustrate the usefulness of the proposed framework for analyzing ERN resilience. The results show that deploying FCSs near the highway entrances and maintaining their operation are relevant factors to enhance the system's resilience. The analysis can provide guidelines to the system operators for effective management of the ERN operation and identify resilience-critical FCSs for system resilience improvement.
\end{abstract}

\section{KEYWORDS:}

System of systems, Resilience, Electric road network, Electric vehicles, Charging stations, Cell transmission model, Dynamic traffic assignment problem, Linear programming

\section{1 | INTRODUCTION}

Electric vehicles and charging infrastructures are being increasingly deployed around the world (Transport \& Environment, 2020), increasing the coupling between the transportation networks and the power grids, bringing new challenges to these systems of systems.
Charging infrastructures play the crucial role of bridging the two systems in a way that they can operate reliably. Naturally, the operational safety of charging infrastructures attracts increasing attention. Burnham et al. (2017) mentioned that the private and secure communication between EVs and the EV service equipment brings the possibility that significant cybersecurity issues could arise with an expansive extreme fast-charging network. Not just individual vehicles or charging stations (CSs) could be influenced by breaches in security, but wide swaths of the transportation system or the power 
grid could also be impacted. Mao, Yuan, Gao, Wang, \& Zhao (2019) discussed the transmission cascading outages induced by ultra-fast PEV charging. Li, Wang, Pu, Wang, \& Zhang (2019) analyzed the characteristics of three types of a malicious attack on the smart Internet of EVs charging network and proposed an association state analysis method on the time series. B. Wang, Dehghanian, Wang, \& Mitolo (2019) evaluated the electrical safety of the large-scale CSs when coupled to renewable power generation considering three failure scenarios: 1) the facility degradation and CS protection failure; 2 ) the cyber-attack between CSs and electric utilities; 3 ) the potential mismatch between the renewable output and CS demand. On the other hand, CSs rely on the supply of electricity from the power grid, and blackouts of this latter are not rare, e.g., the blackouts reported in the U. K. August 2019 (Energy Emergencies Executive Committee, Jan. 2020). Such dependence may cause one or more CSs to be simultaneously unavailable because the connected power grid has failed. Consequently, the inability to supply electricity to EVs might cause perturbations in the increasingly electrified road network (ERN).

The road network is a critical infrastructure, which plays a crucial supporting role in society's functioning. To cope with increasing disruptive events, such as human-made and natural disasters, road network resilience has been paid large attention (Grande, Castillo, Mora, \& LO, 2017). The concept of the resilience of infrastructure systems has different interpretations in the specialized literature (Linkov \& Trump, 2019; Sharma, Tabandeh, \& Gardoni, 2020), such many of them share the general idea that resilience is the ability of a system to prepare for, absorb, recover from, and adapt to disturbances (Linkov et al, 2014). Resilience differs from other concepts like risk, vulnerability, reliability, and robustness. Risk is concerned with the threat of an event to a system and not with the recovery ability (Zhou, Wang, \& Yang, 2019). Vulnerability describes the susceptibility of a system to potential failures and disruptions (Dong, Yu, Farahmand, \& Mostafavi, 2020). Reliability is defined as a system's ability to perform its function for a given time (Galaitsi, Keisler, Trump, \& Linkov, 2021). Robustness is a system's ability to withstand external acute shocks without significant loss of performance (Ayyub, 2014). Resilience describes the system evolution for disruption to recovery. Interested readers can refer to (Ayyub, 2014; Ganin et al, 2016) for more detailed discussions.

Numerous studies (Faturechi \& Miller-Hooks, 2014; Liu \& Song, 2020; X. Zhang, Mahadevan, Sankararaman, \& Goebel, 2018) have been conducted to assess the resilience of the traditional road networks in the context of unexpected natural disasters (i.e., earthquakes and hurricanes) as well as malicious acts. For the resilience analysis of road systems, three main classes of methods are categorized, optimization-based methods, simulation-based methods, and data-driven methods.
Optimization-based methods are typically used to address predefined decision-making objectives. Within these traffic assignment problems, user equilibrium or system optimal (SO) objectives are typically sought for route selection. For example, Nogal, O'Connor, Caulfield, \& Martinez-Pastor (2016) proposed a new dynamic equilibrium-restricted assignment model to minimize the sum of the integrals of travel costs when some links' capacity was reduced because of the road maintenance work. Travel cost and link load were used as resilience indicators to quantify the impacts of the perturbation. Nogal \& Honti (2019) improved this work by incorporating the users' stochastic behavior in the objective function. X. Zhang, Mahadevan, \& Goebel (2019) presented a bilevel mathematical optimization model to reconfigure the network for increasing the transportation system resilience when facing abrupt increases in the traffic demand during emergency evacuations. In this work, the lower-level problem is modeled as a user equilibrium traffic assignment problem, and the upperlevel problem is to minimize the total travel time. The total travel time of all passengers is utilized to quantify the system performance.

For the simulation-based methods, microscopical traffic simulation and graph theory are typically used to simulate changes in transportation network traffic. Ganin et al, (2017) investigated the resilience and efficiency of road networks. A gravity model and percolation theory were used to model the traffic demand and evolution. Efficiency was quantified by the average annual delay per driver, and resilience was assessed by the change in efficiency under natural disasters, which are modeled as a random process and with the likelihood of occurrence proportional to road length. Similar methods for modeling transportation networks and quantifying inefficiency were employed in Ganin et al. (2019). This work assessed the resilience in intelligent transportation systems (ITS), considering malicious attacks (random and targeted disruptions) on intersections and roadways controlled by ITS. Additional delays caused by disruptions were used to estimate the system's resilience. J. Wang, Kong, \& Fu (2019) used the Aimsun microsimulation software to simulate traffic states with vehicle violations on the expressway. The probability of crash was considered with the resilience ability of the expressway.

Unlike the previous two categories of methods, data-driven methods directly take advantage of the historical data instead of considering the system's physical mechanism. With the advancement of technology, monitoring data are, in fact, receiving increasing attention for the resilience assessment of road networks. For instance, Achillopoulou, Mitoulis, Argyroudis, \& Wang (2020) developed a roadmap for monitoringbased quantification of resilience for transport infrastructures, based on a comprehensive review of the current state-ofthe-art. An extended review and evaluation of structural and 
functionality monitoring data are discussed in this work to support resilience assessment of transport infrastructures exposed to natural and human-induced hazards. Social sensing is also being considered to help resilience management for infrastructures. Roy, Hasan, \& Mozumder (2020), and C. Zhang, Yao, Yang, Huang, \& Mostafavil (2020) used social media data to assess disruption types and locations of infrastructures (transportation systems, power systems, etc.) and to analyze the disruptions and corresponding societal impacts following hurricanes.

Data-driven and simulation-based methods are not employed in this study, considering a lack of historical data because of the limited penetration of EVs in current highway systems and the large number of parameters that would need to be calibrated for simulation models. An optimization-based method is adopted here to develop a mathematical electrified traffic model. There are three main challenges that need to be addressed to study the resilience of an ERN: 1) Considering the spatial and temporal nature of EVs, a distributed dynamic traffic model is needed, whereas most existing work is based on static traffic models (Xie, Hu, Wang, \& Chen, 2020). 2) How to accommodate the profiles of CSs and EVs in a traffic model is still a challenge. More specifically, profiles such as the number of chargers in FCSs, driving range (battery capacity), and charging time (remaining state of charge and distance) of EVs have not been considered or precisely characterized in the existing studies. Most of the studies have not considered the battery capacity and state of charge (SoC) of EVs (Chen, Qian, Miao, \& Ukkusuri, 2020); H. Wang, Fang, \& Z10, 2021). This leads to unrealistic solutions in which: EVs might charge multiple times without considering the actual remaining SoC (Lv, Wei, Sun, Chen, \& Zang, 2019); a fixed charging time (charging demand) is assumed for all EVs (Xie et al, 2020); the assigned charging point is beyond the remaining driving range of an EV. 3) Only very few works have been done for the resilience assessment of ERNs, in which existing resilience indicators cannot be directly used since they cannot capture the post-failure function loss of the new components. Therefore, new indicators are needed to capture the global and local impact on an ERN under FCS failures.

To fill the research gaps mentioned above, this paper proposes a framework to assess the resilience of ERNs with extensive use of EVs also for long trips. Within this framework, a linear two-stage dynamic traffic assignment model is proposed to optimize all vehicles' total travel time. The first and second stage models are employed to assign the traffic before and after FCS failures occur, respectively. The proposed model is able to describe the dynamic of EVs and their charging behaviors in FCSs, and therefore captures how local FCS failures impact the ERN performance globally. Different failure scenarios are used as the input parameters of the second stage model to study system resilience. The scenarios include different levels of EV penetration, different failure durations and locations, and pairs of FCSs failings simultaneously.

The proposed framework is based on the cell transmission model (CTM), which describes the traffic flow from the macroscopic level while keeping the microscopic information. CTM is adopted because of the following: 1) CTM is one of the most widely used network loading models due to its simplicity and capability of covering the whole range of traffic dynamics, including queue formation, dissipation, and kinematic waves. 2) CTM is easy to extend and be customized to solve various problems (Venkatraman \& Levin, 2019; Zhu \& Ukkusurl, 2018). For the resilience assessment problem studied in this work, the proposed CTM-based model can be easily extended to consider other details of the charging stations and EVs. This scalability favors the continuation of future work.

CTM for one straightway was first proposed by Daganzo (1994 (1995). Ziliaskopoulos (20)(0) transformed the simulation model into linear programming (LP) one for the single destination system optimal dynamic traffic assignment (SODTA) problem. Doan \& Ukkusuril (2012) solved the holding back issue reported in Ziliaskopoulos work and extended CTM for multiple OD pairs. Zhu \& Ukkusuril (2013) proposed an effortless penalty label method to address the holding back issue under the multiple OD pairs situations. Lo \& Szeto (2002) developed a variational inequality formulation of the dynamic user optimal assignment problem based on the CTM network version. Han, Ukkusuri, \& Doan (2011) proposed complementarity formulations for the CTM-based dynamic user equilibrium with a single O-D pair. Ukkusurı, Han, \& Doan (2012) extended the previous work to a general network version. Mehrabipour, Hajibabai, \& Hajbabaie (2019) presented a decomposition scheme to find near-optimal solutions to CTM-based SO-DTA problems with multiple O-D pairs, with the less computational burden compared to the original solution. Recently, CTM was further improved to address appearing new challenges in the context of the future intelligent transportation system, such as, modeling the proactive driving behavior of connected vehicles (Zhu \& Ukkusuri, 2018), and solving the shared autonomous vehicle routing problem (Venkatraman \& Levin, 2019). However, CTM has been rarely applied in the ERN context for solving the challenges that EVs bring.

The main contribution of the current paper is summarized as follows:

(1) A methodological framework is proposed for the resilience assessment of ERN under FCSs failures, which is of high practical significance in the context of increasingly coupled transportation and power systems but has been rarely studied in the current literature. 
(2) The characteristics of ERN, such as driving range and charging time for EVs and EVs' spatio-temporal nature, have not been well captured in the existing studies. Within the proposed framework, a novel linear programming (LP) model based on CTM is presented to solve the SO-DAT problem integrated with an ERN's characteristics under FCSs normal operation. This model originally defines new components, such as charging cells (links), queueing cells (links), and energy levels, to accommodate the profile of EVs and FCSs in CTM.

(3) To the best of our knowledge, this is the first work that discretizes the battery capacity of an EV and integrates the SO-DTA problem with the profile of EVs and FCSs into a mathematical linear optimization problem.

(4) The LP model is then extended to a two-stage model to capture the system response under different FCS failure scenarios, providing a unified framework for system resilience analysis.

(5) Two metrics, including cumulative throughput of ERN and cumulative utilization of FCSs are developed to quantify the studied system's resilience.

The rest of the article is structured as follows. Section D introduces the CTM and SO-DTA models and the novel model proposed. Section [ 1 presents three metrics for quantifying the impacts of failures of the FCSs on the performance of the transportation network. Section $⿴$ t illustrates a numerical example to show the application of the proposed framework. Finally, Section 5 provides some concluding remarks and future research directions.

\section{2 | METHODOLOGY}

In CTM, a road is divided into homogeneous sections, which are called cells, and the cell length $\left(L_{c}\right)$ is equal to the distance traveled in a free-flow speed $\left(v_{f}\right)$ by a typical vehicle in one time interval $(\tau)$, i.e., $L_{c}=v_{f} \times \tau$. At any time instant $t$, the state of the system is given by the cell occupancy of each cell $x_{i}(t)$. Let $\mathcal{C} / \mathcal{E}$ be the set of cells/links. The core concept of CTM is that the occupancy in cell $i$ at time $t$ equals its occupancy at time $t-1$, plus the inflow and minus the outflow, given by:

$x_{i}(t)=x_{i}(t-1)+\sum_{k \in \Gamma^{-}(i)} y_{k, i}(t-1)-\sum_{j \in \Gamma^{+}(i)} y_{i, j}(t-1), \forall i \in \mathcal{C}, t \in \mathcal{T}$

where $\Gamma^{-}(i) / \Gamma^{+}(i)$ denote the set of predecessor and successor cells of cell $i$ respectively, and $y_{k, i}(t) / y_{i, j}(t)$ represent the vehicles flow along the links from cell $k$ to cell $i$ and from cell $i$ to cell $j$, respectively. Generally, the flow on the link is determined by piecewise linear equation, as follows:

$$
y_{i, j}(t)=\min \left\{x_{i}(t), Q_{i}(t), Q_{j}(t), \delta\left(N_{j}(t)-x_{j}(t)\right)\right\}
$$

where $Q_{i}(t)$ is the in-flow or out-flow capacity of cell $i$ at time t. $N_{i}(t)$ is the maximum number of vehicles that can be present in cell $i$ at time $t . \delta_{i}$ gives the ratio of forward to backward shockwave propagation of the cell $i$. Eq. (II) states that the flow that can advance from cell $i$ to cell $j$ is determined by its own sending capacity $\left(\min \left\{x_{i}(t), Q_{i}(t)\right\}\right)$ and the receiving capacity $\left(\min \left\{Q_{j}(t), \delta_{j}\left(N_{j}(t)-x_{j}(t)\right)\right\}\right)$ of the downstream cell $j$. The sending capacity of cell $i$ is constrained by its own vehicle occupancy and out-flow capacity at time $t$. The receiving capacity of the downstream cell $j$ is constrained by the in-flow capacity and remaining spaces, considering the influence of shock waves of cell $j$.

The existing CTM-based traffic models do not consider the new factors that EVs bring, such as the driving range of EVs and the capacity of FCSs. In this paper, a novel linear programming model for the multiple destinations SO-DTA problem is proposed considering EVs and FCSs (SO-DTA-E\&C) to minimize the total travel cost for all vehicles.

The assumptions in this model are:

(1) There are no backup solutions (such as a backup of the electricity grid system and a buffer of the transformers) to resist failures in charging stations.

(2) Each FCS is only equipped with one type of charger, and these chargers have the same charging speed.

(3) The EV charges as little electricity as possible en-route to make its travel time shortest. The SoC after charged (original SoC plus the charged electricity) should ensure that the EV can reach the destination or the next FCS.

(4) The electricity consumed by an EV is linearly related to the distance traveled. The electricity amount charged by an $\mathrm{EV}$ is linearly related to the charging time. The attributes of different batteries are ignored.

(5) The electricity consumed by the in-vehicle equipment, such as air conditioners and lights, is neglected. When EVs stop, no electricity is consumed.

The first assumption is made based on the aim of this paper. Although the existence of backup electricity can be considered (Galbusera, Giannopoulos, Argyroudis, \& Kakderi, 2018), the purpose of this paper is to investigate the resilience of road networks against FCS failures. This investigation aims to identify the critical components (different importance of FCSs) in the road networks to provide guidance to improve the networks resilience, for example, by identifying at which charging stations the backup electricity should be firstly installed and ensured. Assumption (2) is widely adopted in the literature (Xie et al., 2020) and it also matches the real practice (such 
as Tesla supercharger stations). The third assumption is coherent with the proposed model's objective function, which is to minimize the total travel time. Other phenomena, e.g., the EVs only leave FCSs after being fully charged or $80 \%$ charged, can be easily incorporated by adding constraints on the charging cells. As for the heterogeneous charging preferences of EV drivers, their consideration is not within the scope of this paper. In assumption (4), we assume that the EVs can be replenished the equal energy by the same charger type within the same duration without considering different battery attributes. Similar assumptions or more idealistic assumptions (Chen et al, 2020; Lv et al., 2019; Xie et al,, 2020) that assumed charging demand is fixed for all EVs, is proportional to the link traffic flow, or follow certain probability distributions, can be found in the literature.

In order to track the SoC of EVs, the model accounts for different energy levels $(l)$ to describe the real-time SoC for each EV. Considering EVs with different battery capacities, the average driving range $L_{\text {avg }}$ is used to represent the average battery capacity for all EVs. Although, more categories of battery capacity could be considered by simply extending the current model. However, this would make the model more trivial and computationally expensive without bringing too much added value from a technical or academic perspective. The focus of the present paper is to develop a methodological framework to investigate the resilience of the electrified road network from a system level and, thus, a statistically averaged driving range of all EVs could be acceptable. Additionally, the averaged maximum driving range is also a well acceptable assumption in the literature (Bedogni et al., 2015; Chen et al., 2020). The electricity consumption of EV is assumed to be a linear function of the distance traveled. Therefore, mileage can be used to quantify electrical energy and is used as its unit measurement for simplicity. The battery capacity is discretized into homogeneous energy levels (ELs). Each energy level can supply electric energy for an EV to travel a certain mileage. The mileage traveled by an EV using one energy level is set to be one cell length. Once the cell length is determined, the total number of energy levels $(L)$ of each EV can be calculated by the following equation:

$$
L=\frac{L_{a v g}}{L_{c}}
$$

We use $\mathcal{L}$ to represent the set of all possible energy levels $\{1, \cdots, L\}$. The current EL $(l)$ of an EV represents the remaining distance that can be traveled (i.e., its current SoC). $l \times L_{c} \approx$ the remaining distance that can be traveled $\approx$ current $\mathrm{SoC} \times F$. Driving range $\left(L_{\text {avg }}\right) \approx L \times L_{c} \approx$ full battery $\times F . F$ is the force that an EV overcomes when moving at a constant speed.

In the SO-DTA model, only a single destination is considered. Here, an extended version is introduced to handle a general network with multiple O-D pairs and the route choice

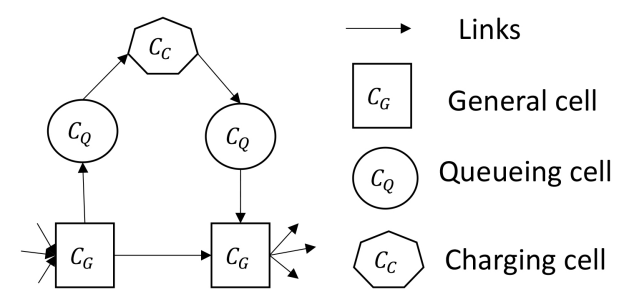

Fig. 1 Cell representation of a charging station.

behaviors are also described. $\mathcal{W}$ is used to represent the set of all O-D pairs. $\mathcal{R}$ represents the set of all the paths. $\mathcal{R}^{w}$ represents the set of the paths belonging to the O-D pair $w$. A path $\left(\mathcal{P}^{r}\right)$ is represented by an ordered collection of cells.

For simplicity, ordinary, merging and diverging cells are called general cells $\left(\mathcal{C}_{G}\right)$, since they share the same update rule. $\mathcal{E}_{D}$ denotes the set of diverging links. In order to integrate the physical component (e.g., FCSs) in the road network into the CTM, charging cells $\left(\mathcal{C}_{C}\right)$ and queueing cells $\left(\mathcal{C}_{Q}\right)$ are defined here. A FCS is modelled by a fixed structure which represents a charging cell sandwiched between two queueing cells, as shown in Fig. 1 .

In SO-DTA-E\&C, charging cells are used to accommodate the vehicles connecting to the chargers. Unlike in CTM, the length of a general cell is defined as the specific physical length of the corresponding road section, whereas the cell length is not applicable to charging cells. In CTM, $N_{i}(t)$ is defined as the maximum number of vehicles that can be present in cell $i$ at time $t$. For charging cell $i, N C_{i}(t)$ is defined, instead, as the maximum number of chargers at time $t$. Additionally, a new parameter $\alpha_{i}^{t}$ is uniquely defined for the charging cell: $\alpha_{i}^{t}$ represents the average charging speed at the FCS $i$ at time $t$, which translates to how many energy levels can be supplied at each charger at station $i$ in a time interval $\tau$. In this paper, the charging capacity refers to the number of chargers $N C_{i}(t)$ and we assume that one FCS in the highway is equipped with the same charger type of same charging speed $\alpha_{i}^{t}$. Generally, EVs charge faster in a commercial FCS than they consume electricity in a common use mode (e.g., no air conditioning) within the same time interval. Hence, under the common use mode, $\alpha_{i}^{t}$ is assumed to be greater or equal to 1 . Moreover, for simplicity, $\alpha_{i}^{t}$ is rounded to an integer. When a FCS fails, $\alpha_{i}^{t}$ is equal to 0 .

Queueing cells are used to accommodate the vehicles waiting to connect to chargers in the FCSs or waiting to leave the FCSs. Such a structure can prevent the charging cell from being congested and can guarantee it works normally when the downstream general cell is congested. The queueing cell before the charging cell can be regarded as the section from the ramp to the parking lot and the one after the charging cell can be regarded as the section from the parking lot to the main road in the service area. Similar to the charging cell, queueing 
cell length is not applicable. $N P_{i}(t)$ is defined as the maximum number of parking spaces for queueing cells. Definitions of parameters for different cells are listed in Tab. $\mathbf{0}$.

Tab. 1 Parameters' definitions for different cells in the extended model

\begin{tabular}{lll}
\hline cells & $L_{c}$ & $N$ \\
\hline Other cells & same to CTM & same to CTM \\
Charging cells & Not applicable & $\begin{array}{l}\text { number of chargers } \\
(N C)\end{array}$ \\
Queueing cells & Not applicable & $\begin{array}{l}\text { number of parking } \\
\text { spaces }(N P)\end{array}$ \\
\hline
\end{tabular}

CTM is proposed based on the hydrodynamic model, which assumes that the flow (velocity) is a function of the density and their relationship follows the triangular fundamental diagram Daganzo (1994 1995). The flow updating equation between cells is derived based on this assumption. Obviously, the relationship among the inflow, velocity and density of EVs at charging cells does not follow the triangular fundamental diagram, since the EVs in charging cells are stationary at most of the time. Therefore, the flow updating into charging cells should be modified. The link beginning with a queueing cell and ending with a charing cell is defined as a charging link $\left(\mathcal{E}_{C}\right)$. The flow updating equation is modified as follows:

$$
\begin{array}{r}
y_{i, j}(t)=\min \left\{x_{i}(t), Q_{i}(t), Q_{j}(t),\right. \\
\left.N C_{j}(t)-x_{j}(t)+y_{j, m}(t)\right\},(i, j) \in \mathcal{E}_{C}, m \in \Gamma_{j}^{+}
\end{array}
$$

The flow $\left(y_{i, j},(i, j) \in \mathcal{E}_{C}\right)$ along the charging link should be constrained by the number of EVs in the queueing cell $\left(x_{i}\right)$ and its out-flow capacity $\left(Q_{i}\right)$, as well as in-flow capacity of the charging cell $\left(Q_{j}\right)$. A relaxed restriction $\left(N C_{j}-x_{j}+y_{j, m}, m \in\right.$ $\left.\Gamma_{j}^{+}\right)$is considered for the charging cell instead of the conservative $\left(\delta \cdot\left(N C_{j}-x_{j}\right)\right)$ used in the CTM. The flow updating equation in CTM assumes that the effects of the outflow is only noticed upstream after some time and, thus, one time step lag exists in CTM. Differently, Eq. (田) assumes that the EVs would flow into charging cells once there are EVs leaving and available chargers appeared, thus, less lags in the modified model, which also helps reduce the time horizon of the model. Since no density waves propagates backwards for the stationary EVs in charging cells, parameter $\delta$ is not needed here.

\section{1 | Formulation of SO-DTA-E\&C}

The proposed SO-DTA-E\&C is able to describe the key features of the FCSs, track the SoC of EVs, and assign the system optimal traffic flow while considering the driving range and the charging time of EVs. The presented SO-DTA-E\&C for the case of normal conditions (no FCS failures) is formulated as below:

The objective of the SO-DTA-E\&C is:

$$
\min _{d, x, \dot{x}, y} \sum_{i \in\left\{C \backslash C_{S}\right\}} \sum_{l \in \mathcal{L}} \sum_{r \in \mathcal{R}} \sum_{t \in 0, \cdots, T_{h}} x_{i}^{l, r}(t)
$$

s.t.

$$
\begin{aligned}
\sum_{r \in \mathcal{R}^{w}} d_{i}^{l, r}(t) & =D_{w}^{l}(t), \forall w \in W, t \in 0, \cdots, T_{d} \\
x_{i}^{l, r}(t) & =x_{i}^{l, r}(t-1)+d_{i}^{l, r}(t-1)-y_{i, j}^{l, r}(t-1),
\end{aligned}
$$

$\forall i \in \mathcal{C}_{R} \cap \mathcal{P}^{r}, j \in \Gamma_{i}^{+}, \forall l \in \mathcal{L}, \forall r \in \mathcal{R}, t \in 1, \cdots, T_{d}+1$

$$
x_{i}^{l, r}(t)=x_{i}^{l, r}(t-1)-\sum_{j \in \Gamma_{i}^{+}} y_{i, j}^{l, r}(t-1),
$$

$\forall i \in \mathcal{C}_{R}, \forall l \in \mathcal{L}, \forall r \in \mathcal{R}, t \in T_{d}+2, \cdots, T_{h}$

$$
x_{i}^{l, r}(t)=x_{i}^{l, r}(t-1)+\sum_{k \in \Gamma_{i}^{-}} y_{k, i}^{l+1, r}(t-1)-\sum_{j \in \Gamma_{i}^{+}} y_{i, j}^{l}(t-1),
$$$$
\forall i \in \mathcal{C}_{G}, \forall l \in\{\mathcal{L}-L\}, \forall r \in \mathcal{R}, \forall t \in \mathcal{T}
$$

$$
x_{i}^{L, r}(t)=x_{i}^{L, r}(t-1)-\sum_{j \in \Gamma_{i}^{+}} y_{i, j}^{L, r}(t-1), \forall i \in \mathcal{C}_{G}, \forall r \in \mathcal{R}, \forall t \in \mathcal{T}
$$

$$
x_{i}^{l, r}(t)=x_{i}^{l, r}(t-1)+y_{k, i}^{l, r}(t-1)-y_{i, j}^{l, r}(t-1),
$$

$\forall k \in \Gamma_{i}^{-}, \forall j \in \Gamma_{i}^{+}, \forall i \in \mathcal{C}_{Q}, \forall l \in \mathcal{L}, \forall r \in \mathcal{R}, \forall t \in \mathcal{T}$

$$
\dot{x}_{i}^{l, r}(t)=x_{i}^{l, r}(t-1)+y_{k, i}^{l, r}(t-1)-y_{i, j}^{l, r}(t-1),
$$

$\forall k \in \Gamma_{i}^{-}, \forall j \in \Gamma_{i}^{+}, \quad \forall i \in \mathcal{C}_{C}, \forall l \in \mathcal{L}, \forall r \in \mathcal{R}, \forall t \in \mathcal{T}$

$$
\begin{gathered}
x_{i}^{L, r}(t)=\sum_{s=0}^{\alpha_{i}^{t}} \dot{x}_{i}^{L-s, r}(t), \quad \forall i \in \mathcal{C}_{C}, \forall r \in \mathcal{R}, \forall t \in \mathcal{T} \\
x_{i}^{l, r}(t)=\dot{x}_{i}^{l-\alpha_{i}^{t}, r}(t), \quad \forall i \in \mathcal{C}_{C}, \forall l \in\left\{\alpha_{i}^{t} \leq l<L\right\}, \quad(11 \mathrm{a}) \\
\forall r \in \mathcal{R}, \forall t \in \mathcal{T}
\end{gathered}
$$

$$
\begin{aligned}
& \sum_{\forall j \in \Gamma_{i}^{+}} \sum_{\forall l \in \mathcal{L}} \sum_{\forall r \in \mathcal{R}} y_{i, j}^{l, r}(t) \leq Q_{i}(t), \forall(i, j) \in \mathcal{E}, \forall t \in \mathcal{T} \\
& \sum_{\forall i \in \Gamma_{j}^{-}} \sum_{\forall r \in \mathcal{R}} \sum_{\forall l \in \mathcal{L}} y_{i, j}^{l, r}(t) \leq Q_{j}(t), \forall(i, j) \in \mathcal{E}, \forall t \in \mathcal{T}
\end{aligned}
$$




$$
\begin{array}{r}
\sum_{\forall i \in \Gamma_{j}^{-}} \sum_{\forall l \in \mathcal{L}} \sum_{\forall r \in \mathcal{R}} y_{i, j}^{l, r}(t)+\delta_{j}(t) \sum_{\forall l \in \mathcal{L}} \sum_{\forall r \in \mathcal{R}} x_{j}^{l, r}(t) \leq \delta_{j}(t) N_{j}(t), \\
\forall j \in \mathcal{C} \backslash \mathcal{C}_{C} \backslash \mathcal{C}_{Q}, \forall t \in \mathcal{T}
\end{array}
$$

$$
\begin{array}{r}
\sum_{\forall i \in \Gamma_{j}^{-}} \sum_{\forall l \in \mathcal{L}} \sum_{\forall r \in \mathcal{R}} y_{i, j}^{l, r}(t)+\sum_{\forall l \in \mathcal{L}} \sum_{\forall r \in \mathcal{R}} x_{j}^{l, r}(t) \leq N P_{j}(t), \\
\forall j \in \mathcal{C}_{Q}, \forall t \in \mathcal{T} \\
\sum_{\forall i \in \Gamma_{j}^{-}} \sum_{\forall l \in \mathcal{L}} \sum_{\forall r \in \mathcal{R}} y_{i, j}^{l, r}(t)+\sum_{\forall l \in \mathcal{L}} \sum_{\forall r \in \mathcal{R}} x_{j}^{l, r}(t)- \\
\sum_{\forall m \in \Gamma_{j}^{+}} \sum_{\forall l \in \mathcal{L}} \sum_{\forall r \in \mathcal{R}} y_{j, m}^{l, r}(t) \leq N C_{j}(t), \forall j \in \mathcal{C}_{C}, \forall t \in \mathcal{T} \\
x_{i}^{l, r}(0)=0, \quad \forall i \in \mathcal{C}, \quad \forall l \in \mathcal{L}, \forall r \in \mathcal{R} \\
y_{i, j}^{1, r}(t)=0, \quad \forall(i, j) \in \mathcal{E}, \forall r \in \mathcal{R}, \forall t \in \mathcal{T}
\end{array}
$$$$
y_{i, j}^{l, r}(t)=0, \quad \forall(i, j) \in \mathcal{E}_{D} \cap(i, j) \notin \mathcal{P}^{r}, \forall r \in \mathcal{R}, \forall t \in \mathcal{T}
$$$$
x_{i}^{l, r}(t) \geq 0, \forall i \in \mathcal{C}, \quad \forall l \in \mathcal{L}, \forall r \in \mathcal{R}, \forall t \in 0, \cdots, T_{h}
$$$$
y_{i, j}^{l, r}(t) \geq 0, \quad \forall(i, j) \in \mathcal{E}, \quad \forall l \in \mathcal{L}, \forall r \in \mathcal{R}, \forall t \in 0, \cdots, T_{h}
$$

where $T_{d}, T_{h}$ and $\mathcal{T}$ are the maximum departure time, maximum time horizon when all EVs have left the network and set of all time intervals up to $T_{h}$, respectively. Considering EVs with different energy levels and destinations, the objective function in Eq. (इ) is modified by adding summations over energy levels and routes. The objective is still to minimize the total travel time over the whole time horizon from a system operator perspective. Eq. (G) guarantees that the traffic demand is satisfied over the departure time period: in each time $t$, the number of departures $d_{i}^{l, r}(t)$ in cell $i$ with energy level $l$ for O$\mathrm{D}$ pair $w$ equals to the traffic demand with that energy level and O-D pair. The source cells can store infinite amounts of traffic. These cells receive traffic directly from the path flow pattern $d_{i}^{l, r}(t)$. In Eq. ([Da), source cells $i$ only belongs to a particular route $r$ of a certain O-D pair $w$. The cell occupancies for all other source cells which do not belong to the route $r$ are equal to zero. Eq. (La) considers the period of departure (from time 0 to $T_{d}$ for variables $\left.d_{i}^{l, r}(t)\right)$ whereas Eq. (ZDD) is for the later period without departures. Constraint (区) expresses the cell mass conservation and gives the specific rules of updating the vehicles occupancies for general cells. Moreover, the pathbased and energy-based occupancy for each cell is tracked. Eq. (区a) states that the current occupancies $x_{i}^{l, r}(t)$ in the general cell $i$ with energy level $l$ at time $t$ equals to its occupancies $x_{i}^{l, r}(t-1)$ minus the outflow $y_{i, j}^{l, r}(t-1)$ with the same energy level $l$, plus the inflow $y_{k, i}^{l+1, r}(t-1)$ with energy level $l+1$, at the last time step. After the EVs flow into the successive cells, their energy levels decline from $l+1$ to $l$. This means that when the EVs stay in the same cell or flow out from a cell, their energy levels do not change. Eq. (8D) details the situation where $l=L$, where $L$ is the upper bound of the energy level (maximum driving range/maximum battery capacity). Since it is not possible that the energy level of an EV is higher than $L$, the term $y_{k, i}^{L+1, r}(t-1)$ is eliminated. Eq. (Q) represents the update rule of the queueing cells while ensuring that no energy is consumed by passing through the cell. This is because the consumed energy from a ramp to a parking lot or from a parking lot to the main road is assumed to be negligible compared to the energy consumption of traveling through a cell length. Eq. (III) states the update rule for charging cells occupancy, where $\dot{x}_{i}^{l, r}(t)$ represents the occupancy of EVs before the SoC of EVs is updated in the charging cell $i$ at time $t$ with energy level $l$. Eqs. (एव) to (एव) state the update rules for energy levels of EVs in the charging cells. Eq. (एव) states that when the energy levels of EVs are between $\left[L-\alpha_{i}^{t}, L\right]$, their energy levels are approximately updated to energy level $L$ after one time period. Eq. (III) states that when energy levels of EVs are between $\left[0, L-\alpha_{i}^{t}\right)$, they increase $\alpha_{i}^{t}$ energy levels after one time period. Eq. (एट) ensures that no EVs' energy levels are below $\alpha_{i}^{t}$ level after being charged for one time period. Note that the charging cell occupancy is conserved before and after the SoC of the EVs is updated, i.e., $\sum_{l} \dot{x}_{i}^{l, r}(t)=\sum_{l} x_{i}^{l, r}(t)$. Eqs. (12a) to (12d) state the specific constraints of determining the flow from cell $i$ to $j$. Eq. (120) states that the flow entering to queueing cells is also constrained by the number of parking spaces remaining $\left(\delta\left(N P_{j}(t)-\sum_{l} \sum_{r} x_{j}^{l, r}(t)\right)\right)$ at time $t$. Eq. (I2t) is equivalent to the fourth term in Eq. (更) and states that the flow that can be received by the charging cells is constrained by the number of available chargers within time interval $t$. Constraint ([13) specifies the initial occupancies. Constraint (펴) ensures that any EV can never exceed its travel range that is no matter when and where, the energy level of an EV must be greater than or equal to 1 . Constraint ([DJ) forces the flow along the links that do not belong to the route $r$ to be zero. Constraints (미) and (피) give the nonnegativity conditions.

\subsection{A two-stage model of the FCS failure process}

Since the occurrence time and location of the failures are random, the second stage model is proposed to solve the traffic assignment problem under uncertain FCS failure conditions. The presented SO-DTA-E\&C model is utilized as the first stage model under FCS normal operations. The solution of the first stage model combined with the information (occurrence 
time, location, duration) of FCS failures are fed into the second stage model as parameters. The information of FCS failures can be generated by appropriate uncertainty models, e.g., models of cascading failures in power grids (Zio \& Aven, 2011); however, this problem is out of the scope of this paper. If we directly use the SO-DTA-E\&C model under FCS failure conditions, it is equivalent to assuming that the system operator is able to foresee when and where the failure occurs, which is unrealistic.

The system state in the normal phase is the same as the system state under the same conditions without failure. Therefore, a corresponding scenario without failures can be assumed, and the system state under this scenario can be calculated by the proposed SO-DTA-E\&C, which is used as the first stage model. The system state in the failure phase plus the recovery phase would be calculated by the second-stage model, which will be introduced later. The scenario results without failures and that of the scenario with failures are denoted as $I$ and $I I$, respectively.

To model the failures of the FCSs, we use $\alpha_{i}^{t}$ to represent when and where the failures occur and for how long the corresponding FCSs do not provide service. Other parameters are the same as for the normal conditions. In practice, failures' location and time of occurrence are usually uncertain, and the system's operator responds to the failures after they occur. Once a failure occurs, the time required to repair is estimated in advance. In this paper, we assume that the system operator immediately receives the failure information (time and location) once it occurs and estimates the time for recovering the charging service. It should be noted that if the repair time can not be estimated in advance, a three-stage model can be implemented without any difficulty. Similarly, the current model can handle multiple simultaneous FCS failures and can be extended into a multi-stage model, the scenarios in which multiple FCSs fail at different locations and time instants.

The two-stage optimization model is formulated. The objective function is the same as the SO-DTA-E\&C model, i.e., the total travel time is minimized (Eq. 18):

$$
\min _{d, x, \dot{x}, y} \sum_{i \in\left\{\mathcal{C} \backslash \mathcal{C}_{S}\right\}} \sum_{l \in \mathcal{L}} \sum_{r \in \mathcal{R}} \sum_{t \in 0, \cdots, T_{h}} x_{i, I I}^{l, r}(t)
$$

$T_{f}$ is the time instant at which the failure occurs. For the normal phase, the system state is calculated by the SO-DTAE\&C model:

$x_{i, I I}^{l, r}(t)=x_{i, I}^{l, r}(t), \forall i \in \mathcal{C}, \forall l \in \mathcal{L}, \forall r \in \mathcal{R}, t \in 0, \cdots, T_{f}-1$

$$
\begin{array}{r}
y_{i, j, I I}^{1, r}(t)=y_{i, j, I}^{1, r}(t), \forall(i, j) \in \mathcal{E}, \quad \forall l \in \mathcal{L}, \forall r \in \mathcal{R}, \\
t \in 0, \cdots, T_{f}-1
\end{array}
$$

$$
\begin{gathered}
d_{i, I I}^{l, r}(t)=d_{i, I}^{l, r}(t), \quad \forall i \in \mathcal{C}_{\mathcal{R}}, \forall l \in \mathcal{L}, \forall r \in \mathcal{R}, \\
t \in 0, \cdots, \min \left\{T_{f}-1, T_{d}\right\} \\
\dot{x}_{i, I I}^{l, r}(t)=\dot{x}_{i, I}^{l, r}(t), \forall i \in \mathcal{C}, \forall l \in \mathcal{L}, \forall r \in \mathcal{R}, t \in 0, \cdots, T_{f}-1
\end{gathered}
$$

Once the failure occurs, the operator receives the information and responds, immediately. All the EVs on the road are dynamically re-routed in response to the failure:

$$
\begin{array}{r}
\sum_{\forall r \in \mathcal{R}^{w} \cap i \in \mathcal{P} r} x_{i, I I}^{l, r}\left(T_{f}\right)=\sum_{\forall r \in \mathcal{R}^{w} \cap i \in \mathcal{P} r} x_{i, I}^{l, r}\left(T_{f}\right), \\
\forall i \in \mathcal{C}, \forall l \in \mathcal{L}, \forall w \in \mathcal{W}
\end{array}
$$

Eq. (23) states that the traffic at cell $i$ is reassigned among these paths which have the same O-D pair and have the same component cell $i$. The traffic demand for all the O-D pairs are conserved.

If only a few chargers fail and the others are still working in one FCS, the time-dependent parameter $N C_{i}(t)$ is used to describe such situation, by adjusting the maximum number of chargers can be used in charging cell $i$ in time interval $t$. The EVs connecting to the failed chargers would go back to the queueing cell and the others keep uninfluenced. Therefore, the nonnegative constraint on the charging links is removed at this moment and the constraint on other cells remains unchanged. In time interval $T_{f}$, Eq. (피) is modified as follows:

$$
y_{i, j}^{l, r}\left(T_{f}\right) \geq 0, \quad \forall(i, j) \in\left\{\mathcal{E} / \mathcal{E}_{C}\right\}, \quad \forall l \in \mathcal{L}, \forall r \in \mathcal{R}
$$

If all chargers fail in one FCS, parameter $\alpha_{i}^{t}$ also can be used to describe such situation. $\alpha_{i}^{t}=0$ means the charging speed of the FCS is 0 (i.e., no electricity supplied) in time interval $t$. If $\alpha_{i}^{t}$ is used, the EVs connecting chargers can stay in FCS waiting for electricity restoration. In FCS fully breakdown situation, the two presentation for failures are equivalent.

After the failures occur, the variables also need to be constrained by the same updating rules in the SO-DTA-E\&C, while the domain of the time is different. Eq. (G) remains unchanged. For updating the EV occupancy of source cells, if $T_{f} \leq T_{d}, \forall t \in T_{f}+1, \cdots, T_{d}+1$ for Eq. (पaa) and Eq. (पb) remain unchanged; if $T_{f}>T_{d}$, Eq. (La) is not needed and $\forall t \in T_{f}+1, \cdots, T_{h}$ for (므). For the cells' occupancy, $t$ is in $T_{f}+1, \cdots, T_{h}$ for Eqs. (ㅈ) - (III) and Eq. (16). For traffic flow, $t$ is in $T_{f}, \cdots, T_{h}$ for Eq. ([2)), Eqs. (녀) - (155). $t$ is in $T_{f}+1, \cdots, T_{h}$ for Eq. (피).

Eqs. (119) to (22) state that the status of the system before the failures occur should be the same as for the normal conditions. After the failures occur, the route choices and energy needed by the EVs that have not departed and have already 
departed are replanned according to the current system's status, informed failures' profiles, and system operation constraints. The response actions include changing the FCSs for some EVs, changing routes for some EVs without charging. Once the failed stations are restored, they are immediately required to provide charging services without delay.

It should be noted that the main focus of this paper is to propose a methodological framework for assessing the resilience of the ERN considering FCS failures. Actually, the SO-DTA problem addressed for developing the framework has already become sufficiently complicated because of the need to consider the driving range, $\mathrm{SoC}$, and the heterogeneous charging time of EVs. Although the user equilibrium (UE) DTA might be more adherent to real life, its use for the framework development would greatly increase the computational time and complexity. The UE-DTA problem without EVs itself is already complicated and computationally expensive. UE-DTA problem solution sets are always nonconvex (Long \& Szeto, 2019) and capturing queue spillback can lead to the nonexistence of UE-DTA solutions (Szeto \& Lo, 2006). To keep the problem tractable, this paper uses the SO principle, based on which the SO-DTA problem can serve as a benchmark for evaluating the performance of the transportation system (Ma, Ban, \& Pang, 2014).

\section{3 | RESILIENCE ASSESSMENT METRICS}

In order to investigate how the FCS failures impact the operation of the system, two perspectives are considered: system performance and local components performance.

Let $\kappa$ indicate a certain FCS failure with certain characteristics. The cumulative throughput performance $\phi^{\kappa}(t)$ is defined to indicate the extent of the delay caused by the failure $\kappa$ for all the EVs by time $t$. The cumulative throughput performance is calculated by the ratio of the actual cumulative arrivals in case of the failure event $\kappa$ to the expected cumulative arrivals in case of no failure by time $t$ :

$$
\phi^{\kappa}(t)=\frac{\sum_{i \in \mathcal{C}_{S}} \sum_{l \in L} \sum_{r \in \mathcal{R}} x_{i, I I}^{l, r}(t)}{\sum_{i \in \mathcal{C}_{S}} \sum_{l \in \mathcal{L}} \sum_{r \in \mathcal{R}} x_{i, I}^{l, r}(t)}
$$

where $x_{i, I}^{l, r}(t) / x_{i, I I}^{l, r}(t)$ is the occupancy in sink cell $i$ with energy level $l$ along route $r$ at time $t$ under FCS normal/failure conditions; $x_{i, I}^{l, r}(t)$ denotes the decision variable $x_{i}^{l, r}(t)$ of the first stage problem presented in Section $2.1 ; x_{i, I I}^{l, r}(t)$ is the decision variable of the second stage problem developed in Section 2.2.

The lower the value of $\phi^{\kappa}(t)$, the more users are delayed and remain on the road by time $t$ and the more performance of the system is lost.
One broadly accepted resilience metric is defined as follows (Nogal \& Honfi, 2019; Ouyang \& Fang, 2017):

$$
R=\frac{\int_{T_{0}}^{T_{h}}[100-P(t)] d t}{T_{h}-T_{0}}
$$

where $T_{0}$ and $T_{h}$ are the initial and end times of the considered time horizon, respectively, and, $P(t)$ is the percentage loss of system performance at time $t$. Eq. (26) measures the resilience by the average performance over the investigated time horizon.

Since the time is discretized in our model, Eq. (즈) is modified and the resilience of the system in terms of the cumulative throughput performance is defined in the following equation:

$$
\chi^{\kappa}=\frac{\sum_{T_{0}}^{T_{h}} \phi^{\kappa}(t)}{T_{h}-T_{0}}
$$

FCSs and routes are the main components in the studied system and their utilizations are calculated, respectively, to explore how the failures impact the operation of these local components. The cumulative utilization of FCS $i, \eta_{i}(t)$, is defined to quantify the contribution of FCS $i$ to EVs charging by time $t$. It is calculated as the ratio of the cumulative energies (energy levels) supplied by FCS $i$ by the same time $t$ to the cumulative energies supplied by all FCSs in the studied system by time $t$. The cumulative utilization $\eta_{i, I}(t)$ of FCS $i$ by time $t$ under no failure conditions is formulated as follows:

$$
\eta_{i, I}(t)=\frac{\sum_{s=0}^{t} \sum_{l \in \mathcal{L}} \sum_{r \in \mathcal{R}} x_{i, I}^{l, r}(s) \cdot \alpha_{i, I}^{s}}{\sum_{s=0}^{t} \sum_{i \in \mathcal{C}_{C}} \sum_{l \in \mathcal{L}} \sum_{r \in \mathcal{R}} x_{i, I}^{l, r}(s) \cdot \alpha_{i, I}^{s}}, \forall i \in \mathcal{C}_{C}
$$

where $\sum_{s=0}^{t} \sum_{l \in \mathcal{L}} \sum_{r \in \mathcal{R}} x_{i, I}^{l, r}(s)$ indicates the summation of EV occupation counts at FCS $i$ in each time interval over the time period from the beginning to the $t$-th time interval. Taking into account the multiplier of the charging speed at FCS $i, \alpha_{i, I}^{s}$, the numerator denotes the cumulative energies (energy levels) supplied by FCS $i$ by time $t$. It should be noted that Eq. (28) does not consider the chargers that are not being used.

The flowchart of the proposed framework is shown in Fig. [ . Within this framework, the input data is mainly composed of three parts: configuration of ERN, traffic demand, and features of FCS failures. For the ERNs, the needed information includes network structures, length, capacity and free-flow speed of roads, location, capacity (number of chargers), and charging speed (charging power) of FCS. The traffic demand includes the origins, destinations, initial states of charge and vehicles' driving ranges. The third part of the data contains locations and durations of FCS failures. In the first stage, the traffic demand and configuration of the ERN are input into the SO-DTA-E\&C model presented in Section 2.$]$. Then, the traffic assignment solution can be obtained for FCS normal conditions. Then, the traffic assignment solutions for the time periods before FCS failure, failure features, post-failure traffic 


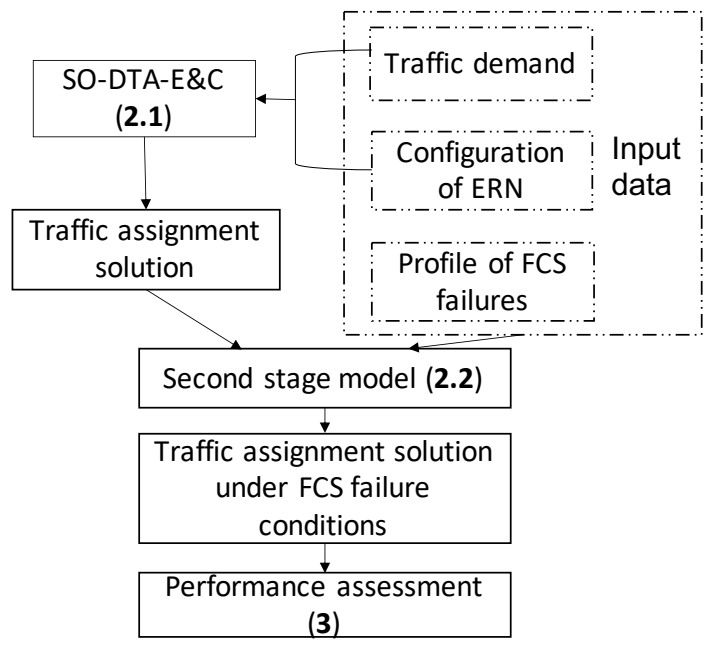

Fig. 2 Flowchart of the proposed framework.

demand, and the studied network configuration are input into the second stage model proposed in Section [2.2. Based on the traffic assignment solutions obtained from the two stages, local components (FCSs) performances and global system resilience can be assessed according to the metrics developed in Section [3.

\section{4 | NUMERIC EXAMPLE}

It should be noted that the proposed model is able to handle scenarios in which few chargers fail to work at one station and the others still work. Such failure scenarios might be more common and usually have low or negligible impacts on the transportation system, i.e., they are so-called high frequency low impact events. On the other hand, from a system resilience perspective, the present paper focuses on low-frequency high impact events, where an entire FCS fails, or several FCSs fail simultaneously. These events, although with relatively low likelihood, can occur in extreme cases such as power blackouts and extreme weather events. From a global risk perspective (Franco et al, 2020), it is reported that extreme weather events (e.g., floods, storms) and natural disasters (e.g., earthquakes, tsunamis) dominate (1st and 3rd) in terms of likelihoods and impacts (rank 5th and 6th). Cyberattacks and breakdowns of critical information infrastructures have similar impacts but lower likelihoods compared to natural disasters. Therefore, it is of high practical significance to study these high-impact events to help identify the worst-consequence scenario and manage the resilience of the studied system.

\section{1 | Data description}

The network from (Nguyen \& Dupuis, 1984) is adopted here, since it is a representative network and widely employed in literature (Long \& Szeto, 2019; Ukkusuri et al, 2012; X. Zhang \& Mahadevan, 2017). Although the size is not large, the structure is complex enough to illustrate the proposed framework. It is modified here by adding 4 FCSs. The FCSs are assumed to be only equipped with DC fast chargers and 20 chargers are installed at each station. They provide charging through $480 \mathrm{~V} \mathrm{AC}$ input. They can deliver 60 to 80 miles of range in 20 minutes of charging (U.S. Department of Energy, 2019).

We take the time step $\tau=6 \mathrm{~min}$ and the free-flow speed $v_{f}=50 \mathrm{miles} / \mathrm{h}$. In 2018, the median of the EV distance ranges on full charge is estimated to be 125 miles, as reported by the U.S. Department of Energy (2020). This data is used as the average maximum driving range of EVs in this study. Therefore, the total energy levels amount to 25 levels using Eq. (B)). The charging power for each charger is $60-80$ miles per 20 minutes, i.e., with a charging speed of $3.6-4.8$ energy levels per time period. For simplicity, we set $\alpha_{i, I}^{t}=4$ energy levels per time period in normal conditions. All used data and parameters are uploaded to Supplementary material (H. Wang, Abdin, Fang, \& Zio, 2021).

\subsection{1 | Initial energy levels}

In this paper, we distinguish the vehicles by whether they have charging demands en-route rather than their motor types (gasoline-powered or electricity powered), since the EVs without the charging demands en-route have the same route choices behavior as the gasoline-powered vehicles. Since the objective function is to minimize the total travel time of all vehicles, the vehicles with and without charging demands en-route cooperatively use the limited resources of both road capacities and charging facilities and, thus, no competitive behaviors among them.

EV penetration levels are investigated in this paper, which is modeled by the initial energy levels (IELs) of EVs. These EVs with high initial energy levels have no charging demands during their trips, then can be regarded as non-EVs. In order to systematically investigate the impacts of different $\mathrm{EV}$ penetration levels, we conduct the proposed method on 5 scenarios with different IEL settings under a certain failure event. The IELs of EVs are mapped into 6 sets: Very Low (VL), low (L), medium $(\mathrm{M})$, high $(\mathrm{H})$ and very high $(\mathrm{VH})$. Suppose an EV has an IEL $L_{I}$ and needs to travel from A to B. $L_{S P} / L_{L P}$ is the required IEL for completing the shortest/longest path between A and B. $L_{N F C S} / L_{F F C S}$ is the required IEL for arriving at the nearest FCS (NFCS)/farthest FCS (FFCS) from A, which is less than or equal to $L_{S P}$. Their relationships is illustrated in Fig. [3. When we say the required IEL, we only consider 


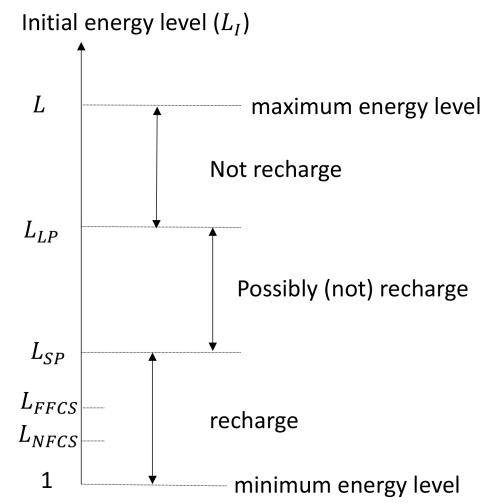

Fig. 3 The range of the initial energy level.

the energy levels consumed by traveling the physical distance from the origin to the certain place and the time-dependent energy consuming is not considered for the moment. Set VL includes the IELs of EVs, which can not support EVs to arrive at the NFCS from the origin, $\mathrm{VL}=\left\{L_{I}<L_{S P}\right\}$. If the IELs allow EVs to reach between the NFCS and the farthest FFCS, the IELs belongs to set $\mathrm{L}, \mathrm{L}=\left\{L_{N F C S} \leq L_{I} \leq L_{N F C S}\right\}$. Set $M$ includes the IELs allowing EVs to reach between the FFCS and the destination by the SP, $\mathrm{M}=\left\{L_{N F C S}<L_{I} \leq L_{S P}\right\}$. Set $\mathrm{H}$ includes the IELs allowing EVs to reach the destination, $\mathrm{H}=\left\{L_{S P}<L_{I} \leq L_{L P}\right\}$. The rest of IELs allowing EVs to reach the destination and left extra electricity, belong to $\mathrm{VH}$, $\mathrm{VH}=\left\{L_{I}>L\right\}$. If IELs of EVs are in the set VH, these EVs can be regarded as non-EVs since they do not have to charge en-route, no matter which route they choose. The specific intervals of energy levels for each set and O-D pair are listed in Tab. घ.

The IEL $L_{S P}$ for completing the SP determines the minimum upper bound of the IEL of the EV that needs to be recharged en-route. The IEL $L_{L P}$ for completing the LP determines the maximum lower bound of the IEL of the EV that it does not need to be recharged en-route. Once $L_{I}<E_{S P}$, then the EV must be recharged on the way. If $L_{I} \geq L_{S P}$, whether the EV needs to be recharged en-route is decided by which route it will take.

Tab. 2 Intervals of energy levels for each set and O-D pairs

\begin{tabular}{cccccc}
\hline O-D & Very Low & Low & Medium & High & Very High \\
\hline & $<L_{N F C S}$ & $\leq L_{F F C S}$ & $\leq L_{S P}$ & $\leq L_{L P}$ & $>L_{L P}$ \\
$1-10$ & {$[1,4]$} & {$[5,6]$} & {$[7,10]$} & {$[11,13]$} & {$[14,25]$} \\
$1-29$ & {$[1,4]$} & {$[5,10]$} & {$[11]$} & {$[12,14]$} & {$[15,25]$} \\
$11-10$ & {$[1,3]$} & {$[4,8]$} & {$[10,11]$} & {$[12]$} & {$[13,25]$} \\
$11-29$ & {$[1,3]$} & {$[4,8]$} & {$[9]$} & {$[10,13]$} & {$[14,25]$} \\
\hline
\end{tabular}

The percentage distributions of the IELs for the 5 scenarios are shown in Tab. [3 . In all scenarios, we assume that there are 100 departures for each O-D pair at each time step and the distribution of the IELs of EVs remains the same within the maximum departure time. The 5 scenarios can be regarded as different EV penetration levels, ranging from at least $90 \%$ to at least $50 \%$ of vehicles being not EVs.

The certain failure event $\kappa_{b}$ is assumed to occur at the 10th time step and to be restored after one hour (at the 20th time step) for FCS 41. This failure event can be modelled in the second stage model by changing the value of parameter $\alpha_{i, I I}^{t}$, which denotes the charging speed at FCS 41 at time $t$ in the second stage model. To model failure event $\kappa_{b}, \alpha_{41, I I}^{t}$ is set to 4 for $t \in\{0, \cdots, 9\}$, meaning that each charger at FCS 41 in each time interval can supply 4 energy levels from time 0 to $9 ; \alpha_{41, I I}^{t}, t \in\{10, \cdots, 19\}$ is set to 0 , indicating that all the chargers at FCS 41 are failed from time intervals 10 to 19, and cannot provide charging service during this period; $\alpha_{41, I I}^{t}$ is set to 4 again for the rest time horizon to represent the service restoration of the chargers.

Tab. 3 Distribution of IELs for each scenario

\begin{tabular}{cccccc}
\hline Scenarios & VL & L & M & H & VH \\
\hline$S_{1}$ & $0 \%$ & $3 \%$ & $3 \%$ & $4 \%$ & $90 \%$ \\
$S_{2}$ & $0 \%$ & $6 \%$ & $6 \%$ & $8 \%$ & $80 \%$ \\
$S_{3}$ & $0 \%$ & $10 \%$ & $10 \%$ & $10 \%$ & $70 \%$ \\
$S_{4}$ & $0 \%$ & $13 \%$ & $13 \%$ & $14 \%$ & $60 \%$ \\
$S_{5}$ & $0 \%$ & $16 \%$ & $16 \%$ & $18 \%$ & $50 \%$ \\
\hline
\end{tabular}

\subsubsection{Characteristics of the failure}

In order to illustrate the proposed model and explore how the system performs under various scenarios, the failures in FCSs have to be characterized. In this section, two main characteristics of the failures are considered: duration and location. In this section, let the settings of IELs in all scenarios follow the $S_{2}$ and failure event $\kappa_{b}$ as a reference to other scenarios. The failure occurrence time in all scenarios start at the 10th time step.

Duration of the failure varies from half an hour (5 time steps) to two hours (20 time steps), while the failure location is kept at No. 41 , which is denoted as $\kappa_{d 5}, \kappa_{d 15}, \kappa_{d 20}$, respectively. Assume the failure duration is one hour and the failure is located at each FCS in the studied network, which is denoted as $\kappa_{l 38}, \kappa_{l 44}$, and $\kappa_{l 47}$, respectively. If two or more FCSs are connected to a same power grid, once outages occur in this power grid that might cause these FCSs to become unavailable, simultaneously. Thus, we simply investigate scenarios which are permutations of two FCSs in the studied network failing simultaneously. The specific profile of each failure is listed in Tabs. 团 and [ . 
Tab. 4 The profile of the failure in each scenario

\begin{tabular}{|c|c|c|c|c|c|c|c|}
\hline \multirow{2}{*}{ Failures } & \multicolumn{3}{|c|}{ Duration (time steps) } & \multirow[b]{2}{*}{$\frac{\kappa_{b}}{10}$} & \multicolumn{3}{|c|}{ Location } \\
\hline & $\kappa_{d 5}$ & $\kappa_{d 15}$ & $\frac{\kappa_{d 20}}{20}$ & & $\frac{\kappa_{147}}{10}$ & $\kappa_{138}$ & $\frac{\kappa_{144}}{10}$ \\
\hline & 41 & 41 & 41 & 41 & 47 & 38 & 44 \\
\hline
\end{tabular}

\section{2 | Results and analysis}

\subsection{1 | Cumulative throughput performance}

Figs. 1 and $\square$ show the total travel time by all users and the system resilience under different scenarios, respectively. The obtained results allow for an intuitive explanation. For example, we observe that the distributions of the objective values and of the resilience values have a opposite trend; Figs. [ and [ show that more EVs with lower IELs (higher EV penetration) cause lower resilience values and higher objective values; longer failure duration leads to lower resilience and higher objective values; resilience and objective value vary of different failure locations. Generally, two FCSs failing simultaneously cause more performance loss and longer total travel time than one FCS failure.

Additionally, Fig. 6(a) shows that as EV penetration increases, the total travel time of the entire system increases steadily, i.e., users expect to take a longer time to reach their

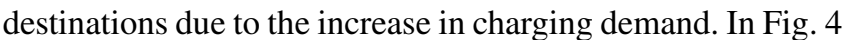
under failure event $\kappa_{b}$, when the EV penetration rate increases from $10 \%$ to $20 \%$, the system resilience in terms of cumulative throughput is reduced the most $(0.3 \%)$. As EV penetration further increases, the marginal reduction in system resilience becomes smaller. This is probably due to the fact that the impact of FCS 41 failure $\left(\kappa_{b}\right)$ on system resilience is limited by its service capacity.

Comparing among different scenarios, we could find that FCS 41 plays an extremely important role in this studied network. The system performance loss of FCS 41 failure for 10 time steps is more than that of FCS 47 and 38 failing simultaneously for 10 time steps. The system performance loss of FCS 41 failure for 15 time steps is even more than that of the combination of any two FCSs failing simultaneously for 10 time steps. We discuss why FCS 41 is extremely important in Section 4.2 .2

Figs. 6 (a) - 6 (d) show the system performance evolution over time under different scenarios. We can observe that the changes in system performance lag behind the failure changes. This is because the system performance is calculated based on the number of EVs in the sink cell, while the delayed EVs need time to arrive at the sink cell from the failure location. Therefore, system performance would not be impacted until the influenced EVs arrive at the sink cell. As shown in Fig. 6 (a), the system performance may increase temporarily, but it will eventually oscillate down because of the failure. Additionally,

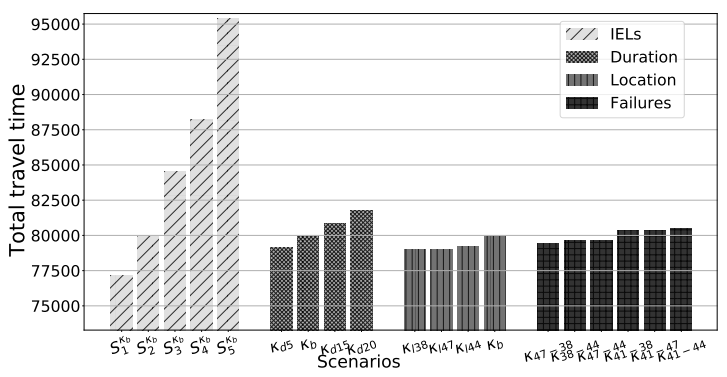

Fig. 4 Objective values under different scenarios

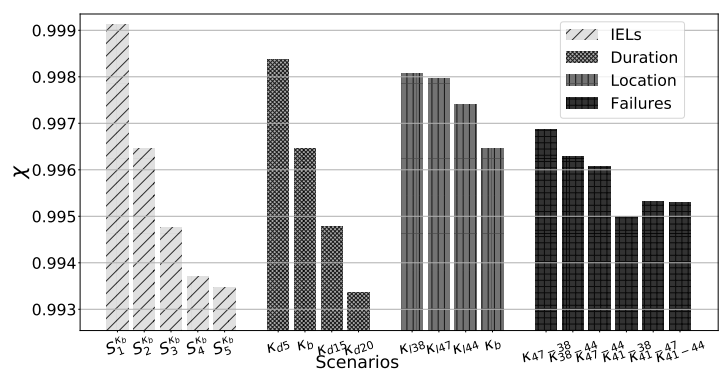

Fig. 5 Resilience under different scenarios

it can be found that the system performance needs longer time to recover when there are more EVs with lower IELs (higher $\mathrm{EV}$ penetration). This is because such failure causes more $\mathrm{EVs}$ to be delayed. Fig. 6 (b) shows that the longer failure duration would result in the system performance decreasing to a worse point. Fig. 6(c) shows that the system performance variation under failure $\kappa_{b}$ lags behind other scenarios. This is because FCS 41 is farthest to the destinations. As shown in Fig. 6 (d), when the failure scenarios involve No. 41, the system needs a longer time to recover.

\subsubsection{Cumulative utilization of FCSs}

Fig. $\square$ shows the evolution of the cumulative utilization of FCSs over time under different settings of IELs shown in Tab. [B] . $S_{2}$ is the same to Fig. 8 (b). $S_{4}$ is omitted here, since the cumulative utilization of FCSs under such a scenario has similar trends to that shown in Figs. 7 (b) and 7 (c). The the same colors denote the same FCS. The lines and the circles lines denote the FCS normal and failure conditions, respectively.

As shown in Fig. 1 (a), after failure occurs, the cumulative utilization of FCS 41 immediately decreases and the cumulative utilization of other FCSs increase to varying degrees. After the failed FCS is restored, the cumulative utilization of FCS 41 gradually increases but is still less than that of the normal conditions. It means some EVs change the routes and chose to charge in other FCSs because of the failure. As in Fig. Z , FCS 41 contributes always the most charging service among all the 
Tab. 5 The profile of the failure in each scenario

\begin{tabular}{ccccccc}
\hline Failures & $\kappa_{41-47}$ & $\kappa_{41-38}$ & $\kappa_{41-44}$ & $\kappa_{47-38}$ & $\kappa_{47-44}$ & $\kappa_{38-44}$ \\
\hline Duration (time steps) & 10 & 10 & 10 & 10 & 10 & 10 \\
Location & $41 \& 47$ & $41 \& 38$ & $41 \& 44$ & $47 \& 38$ & $47 \& 44$ & $38 \& 44$ \\
\hline
\end{tabular}

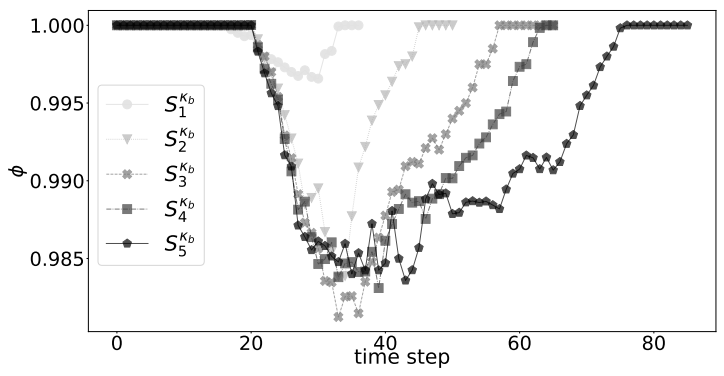

(a) Evolution of system performance under different IELs

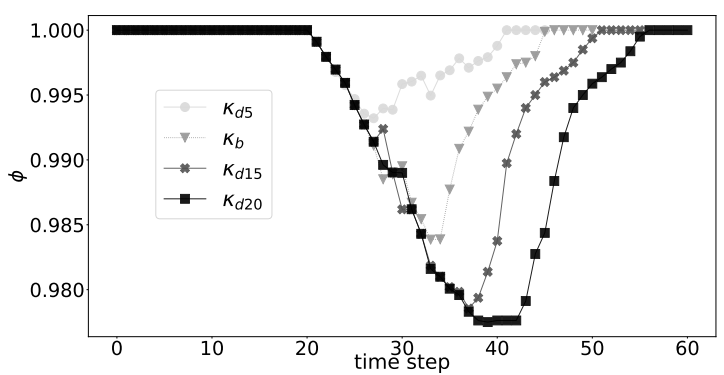

(b) Evolution of system performance under different failure durations

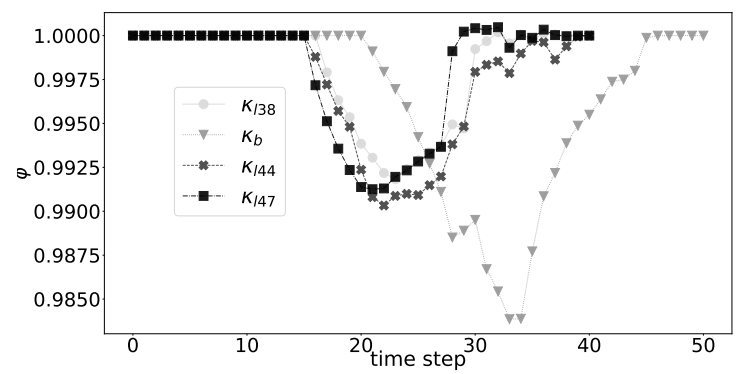

(c) Evolution of system performance under different failure locations

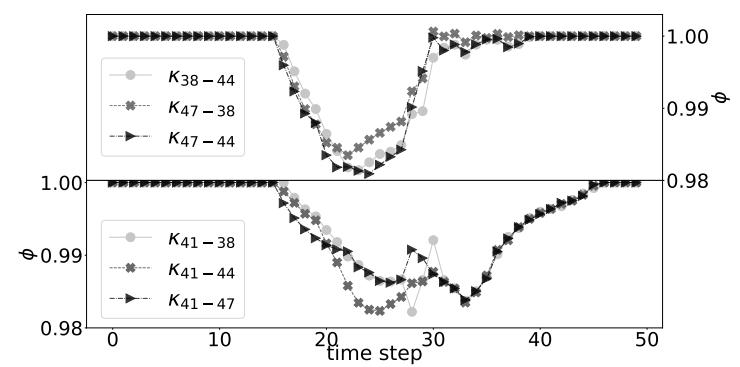

(d) Evolution of system performance under different failure combinations
FCSs in all studied scenarios. This is because FCS 41 occupies an important position in the topological structure of the network. Firstly, the FCS is deployed at an intersection where there is more traffic flow. Secondly, FCS 41 is the nearest FCS both from source cells 1 and 11, meaning that only FCS 41 can provide services for EVs with low IEL departing from the two source cells. Once FCS 41 fails, these EVs with low IEL can only wait until it recovers, since their remaining energies are not enough to support them to reach the next FCS. This result implies that it might be beneficial to deploy FCSs close to city gate entrances of highways to provide much-needed charging services for vehicles with low IEL. The reliable operation of these FCSs is also important to enhance the global resilience of the ERN.

Different distributions of IELs of EVs make the ranking of cumulative utilization different. For example, the cumulative utilization of FCS 44 is the lowest in $S_{1}$, whereas its cumulative utilization increases to the second place in the rest scenarios, as shown in Figs. T(a) - T(c), This is because FCS44 locates at the road section shared by all O-D pairs in the network. Thus, both FCS 41 and 44 can serve more EVs when EV penetration increases, comparing to FCS 38 and 47 that only serve 2 O-D pairs. We also can observe that the cumulative utilization difference of FCSs between the normal and failure conditions becomes smaller with more EVs equipped by lower initial energy levels (higher EV penetration). More EVs with lower initial energy levels would make FCSs in the studied network busier. When a FCS fails, more influenced EVs could not change their charging place to reduce the total travel time, since other FCSs are also very busy. Even if they drive to other FCSs, this would not save much time for them because of extra driving and waiting time needed. Therefore, the performance of the system decreases with increasing EV penetration.

Figs. 8 (a) - 8 (d) show the cumulative utilization evolution of each FCS over time under the scenarios that the failure occurs in different FCSs. When FCS 38, 41 and 47 fails, respectively, cumulative utilization of FCS 44 always absorbs the influence of the failure most among all FCSs, because of its advantageous location, although the increased degree varies with the scenarios.

Fig. 6 Evolution of system performance under different scenarios 


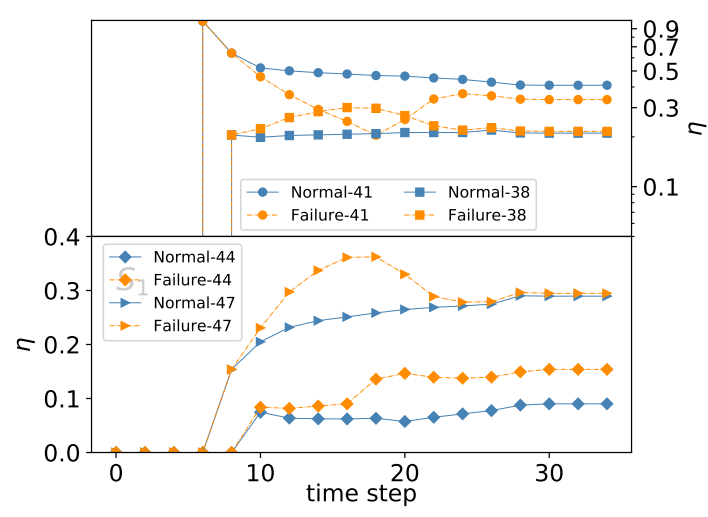

(a) cumulative utilization of each CS under Scenario $S_{1}$

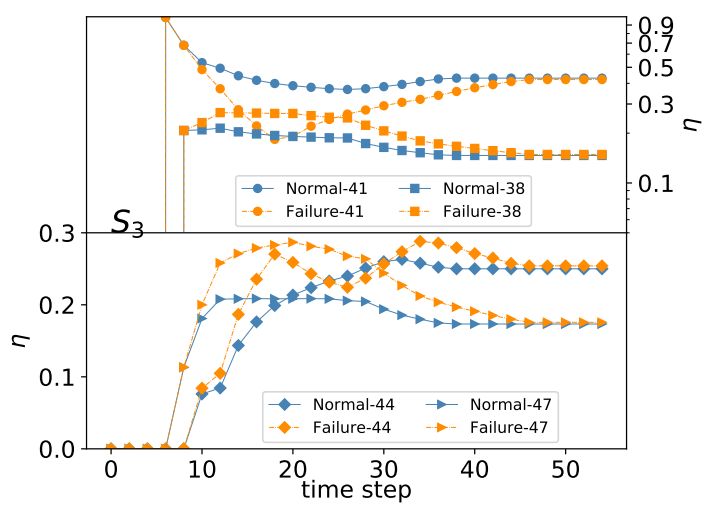

(b) cumulative utilization of each CS under Scenario $S_{3}$

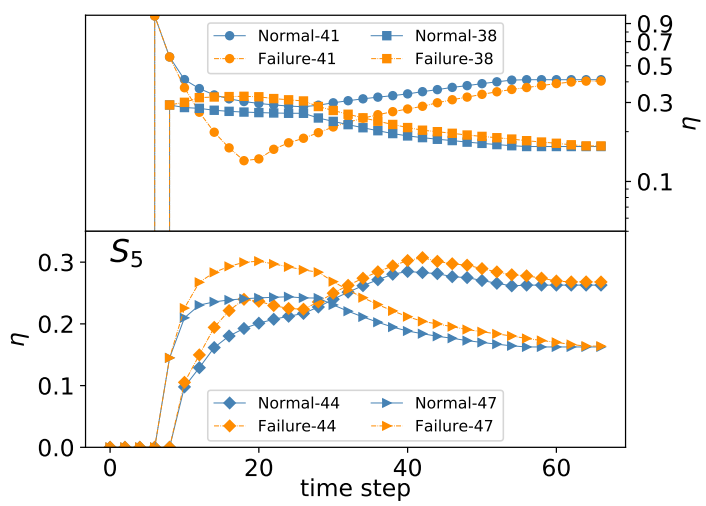

(c) cumulative utilization of each CS under Scenario $S_{5}$

Fig. 7 Evolution of cumulative utilization of each CS over time under different initial energy levels settings.

\section{5 | CONCLUSIONS}

This paper proposes a framework to assess the resilience of the electrified road network (ERN) considering failures in fast-charging stations (FCSs), which are rarely considered in

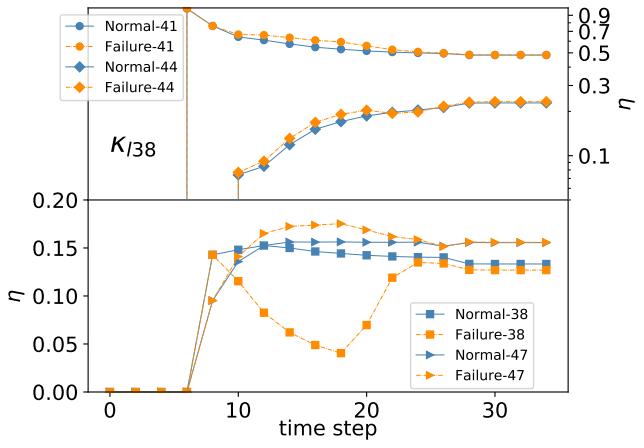

(a) cumulative utilization evolution when CS 38 fails

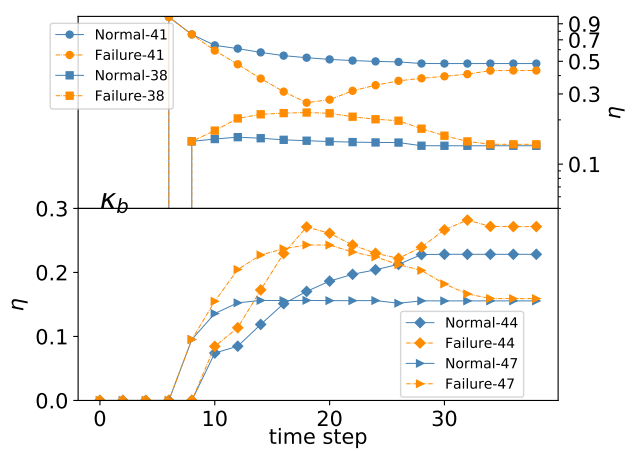

(b) cumulative utilization evolution when CS 41 fails

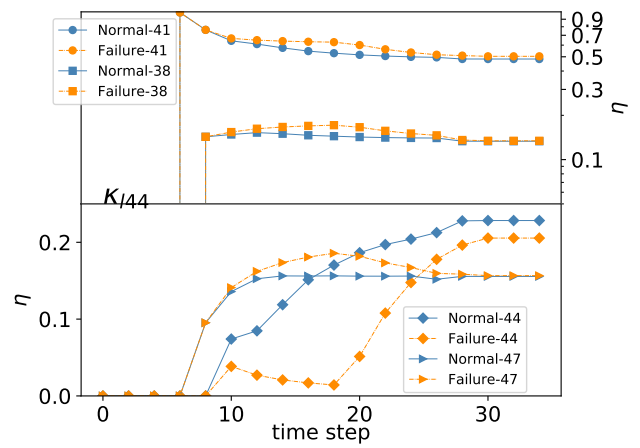

(c) cumulative utilization evolution when CS 44 fails

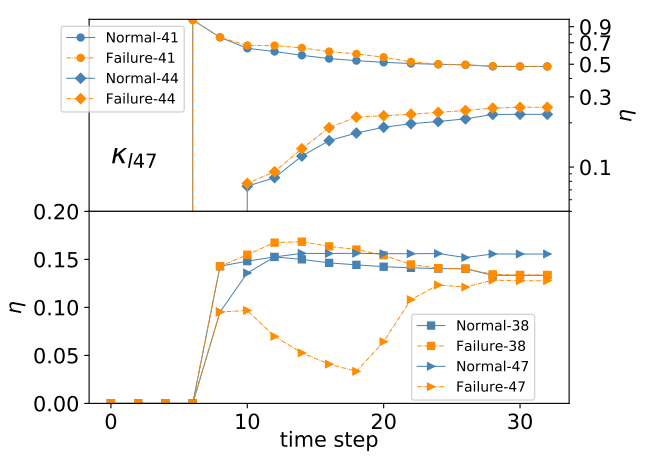

(d) cumulative utilization evolution when CS 47 fails

Fig. 8 Evolution of cumulative utilization of each CS over time under scenarios with different failure locations 
this context. Within this framework, a novel linear programming model based on the cell transmission model (CTM) is developed to estimate the system states by solving the system optimal dynamic traffic assignment model. This model originally contains new factors that EVs bring in CTM, such as charging cells (links), queueing cells (links), and energy levels, so that the profiles of FCSs, driving ranges of electric vehicles (EVs), and state of charge (SoC) of EVs at each time step can be described. Considering the potential failures in the FCSs, a two-stage model is further proposed considering the uncertain nature of failures. Three metrics are presented for assessing the resilience of the ERN and the impact of failures on the FCSs. A numerical example is studied to illustrate the effectiveness of the proposed framework in assessing ERN resilience. The results show that the resilience of an electrified transportation system, quantified by the cumulative traffic throughput, is highly affected by the penetration level of EV, as well as the intensity of the FCS failures (i.e., failure duration, number of simultaneously failed FCSs). More EVs with lower initial energy levels (higher EV penetration), longer failure duration, and two failures occurring simultaneously could generally cause lower system resilience in terms of cumulative throughput performance. The FCS (No. 41) nearest to the highway network's entrances has the highest utilization. Thus, it could cause severe loss of the system performance once it fails. The FCS (No. 44) on the shared road sections by multiple destinations could absorb more impacts of the failures when other FCSs fail.

The result implies that deployed FCSs at the highway entrance could provide much-needed charging services for owners who forget to charge before their trips. The normal operation of these FCSs is also critical, which helps to enhance the ERN's resilience. The results showed the reduction of the cumulative performance remains within $2 \%$ before the system is recovered. The reason is that the studied network is robust to the considered failure scenarios. Indeed, the deployment of FCSs is dense, and the number of chargers in each FCS is also relatively large in the studied ERN.

The results of the proposed two-stage model could be used as a benchmark to analyze the spatial-temporal traffic distribution and charging demand. The proposed framework allows us to identify the critical components (different importance of FCSs) for ERN resilience. Such quantitative information could provide guidance to operators to improve the system resilience against FCS failures, e.g., by identifying at which FCS the backup electricity should be primarily installed against potential power blackout. The computational times are influenced by many factors, such as the maximum time horizon, traffic demand, topology of the ERN, configuration of the FCSs, EV penetrations and their battery capacities. It should be noted that the proposed models are LPs, which can be solved very efficiently using standard polynomial time algorithms. Moreover, computational time analysis is not the main interest of this paper. For more detailed discussions, please refer to the Supplementary Material (H. Wang, Abdin, et al., 202I).

Other topics for further research include user equilibrium conditions, optimal allocation of FCSs considering constraints from the electric distribution system and the ERN, and uncertainties in traffic demand. The proposed model framework can be easily extended to study other disruptive scenarios like road damages caused by extreme weather events leading to inaccessibility of the FCSs. This is interesting for future extensions of the modeling framework. Future studies could investigate the uncertainties of FCS failure events by considering their likelihoods of occurrence and the possible duration and location. For example, this can be based on an empirical analysis of historical data. It is a promising direction to improve the efficiency of the current model by cooperating with the link transmission model (Long \& Szeto, 2019). It is also promising to employ queue-model-based or simulation methods to solve the user equilibrium dynamic traffic assignment problem in the context of the ERN.

\section{ACKNOWLEDGMENTS}

The participation of Hongping Wang to this research is supported by China Scholarship Council (No. 201606990003). The participation of Enrico Zio to this research is supported by the Energy for Motion project of the Department of Energy of Politecnico di Milano, funded by the Italian Ministry of University and Research (MUR) through the Department of Excellence grant 2018-2022.

\section{References}

Achillopoulou, D. V., Mitoulis, S. A., Argyroudis, S. A., \& Wang, Y. (2020). Monitoring of transport infrastructure exposed to multiple hazards: a roadmap for building resilience. Science of the total environment, 141001.

Ayyub, B. M. (2014). Systems resilience for multihazard environments: Definition, metrics, and valuation for decision making. Risk analysis, 34(2), 340-355.

Bedogni, L., Bononi, L., Di Felice, M., D’Elia, A., Mock, R., Morandi, F., ... Vergari, F. (2015). An integrated simulation framework to model electric vehicle operations and services. IEEE Transactions on vehicular Technology, 65(8), 5900-5917.

Burnham, A., Dufek, E. J., Stephens, T., Francfort, J., Michelbacher, C., Carlson, R. B., ... others (2017). Enabling fast charging-infrastructure and economic considerations. Journal of Power Sources, 367, 237-249. 
Chen, R., Qian, X., Miao, L., \& Ukkusuri, S. V. (2020). Optimal charging facility location and capacity for electric vehicles considering route choice and charging time equilibrium. Computers \& Operations Research, 113, 104776.

Daganzo, C. F. (1994). The cell transmission model: A dynamic representation of highway traffic consistent with the hydrodynamic theory. Transportation Research Part B: Methodological, 28(4), 269-287.

Daganzo, C. F. (1995). The cell transmission model, part II: network traffic. Transportation Research Part B: Methodological, 29(2), 79-93.

Doan, K., \& Ukkusuri, S. V. (2012). On the holding-back problem in the cell transmission based dynamic traffic assignment models. Transportation Research Part B: Methodological, 46(9), 1218-1238.

Dong, S., Yu, T., Farahmand, H., \& Mostafavi, A. (2020). Bayesian modeling of flood control networks for failure cascade characterization and vulnerability assessment. Computer-Aided Civil and Infrastructure Engineering, 35(7), 668-684.

Energy Emergencies Executive Committee. (Jan. 2020). Gb power system disruption on 9 august 2019 energy emergencies executive committee (e3c): Final report (Tech. Rep.). Department for Business, Energy \& Industrial Strategy.

Faturechi, R., \& Miller-Hooks, E. (2014). A mathematical framework for quantifying and optimizing protective actions for civil infrastructure systems. Computer-Aided Civil and Infrastructure Engineering, 29(8), 572-589.

Franco, E., et al. (2020). The global risks report 2020. In World economic forum.

Galaitsi, S., Keisler, J. M., Trump, B. D., \& Linkov, I. (2021). The need to reconcile concepts that characterize systems facing threats. Risk Analysis, 41(1), 3-15.

Galbusera, L., Giannopoulos, G., Argyroudis, S., \& Kakderi, K. (2018). A boolean networks approach to modeling and resilience analysis of interdependent critical infrastructures. Computer-Aided Civil and Infrastructure Engineering, 33(12), 1041-1055.

Ganin, A. A., Kitsak, M., Marchese, D., Keisler, J. M., Seager, T., \& Linkov, I. (2017). Resilience and efficiency in transportation networks. Science advances, 3(12), e1701079.

Ganin, A. A., Massaro, E., Gutfraind, A., Steen, N., Keisler, J. M., Kott, A., ... Linkov, I. (2016). Operational resilience: concepts, design and analysis. Scientific reports, $6(1), 1-12$.

Ganin, A. A., Mersky, A. C., Jin, A. S., Kitsak, M., Keisler, J. M., \& Linkov, I. (2019). Resilience in intelligent transportation systems (its). Transportation Research Part C: Emerging Technologies, 100, 318-329.

Grande, Z., Castillo, E., Mora, E., \& Lo, H. K. (2017).
Highway and road probabilistic safety assessment based on bayesian network models. Computer-Aided Civil and Infrastructure Engineering, 32(5), 379-396.

Han, L., Ukkusuri, S., \& Doan, K. (2011). Complementarity formulations for the cell transmission model based dynamic user equilibrium with departure time choice, elastic demand and user heterogeneity. Transportation Research Part B: Methodological, 45(10), 1749-1767.

Li, Q., Wang, Y., Pu, Z., Wang, S., \& Zhang, W. (2019). Time series association state analysis method for attacks on the smart internet of electric vehicle charging network. Transportation Research Record, 2673(4), 217-228.

Linkov, I., Bridges, T., Creutzig, F., Decker, J., Fox-Lent, C., Kröger, W., ... others (2014). Changing the resilience paradigm. Nature Climate Change, 4(6), 407-409.

Linkov, I., \& Trump, B. D. (2019). The science and practice of resilience. Springer.

Liu, W., \& Song, Z. (2020). Review of studies on the resilience of urban critical infrastructure networks. Reliability Engineering \& System Safety, 193, 106617.

Lo, H. K., \& Szeto, W. Y. (2002). A cell-based variational inequality formulation of the dynamic user optimal assignment problem. Transportation Research Part B: Methodological, 36(5), 421-443.

Long, J., \& Szeto, W. Y. (2019). Link-based system optimum dynamic traffic assignment problems in general networks. Operations Research, 67(1), 167-182.

Lv, S., Wei, Z., Sun, G., Chen, S., \& Zang, H. (2019). Optimal power and semi-dynamic traffic flow in urban electrified transportation networks. IEEE Transactions on Smart Grid, 11(3), 1854-1865.

Ma, R., Ban, X. J., \& Pang, J.-S. (2014). Continuous-time dynamic system optimum for single-destination traffic networks with queue spillbacks. Transportation Research Part B: Methodological, 68, 98-122.

Mao, D., Yuan, C., Gao, Z., Wang, J., \& Zhao, R. (2019). Online prediction for transmission cascading outages induced by ultrafast PEV charging. IEEE Transactions on Transportation Electrification, 5(4), 1124-1133.

Mehrabipour, M., Hajibabai, L., \& Hajbabaie, A. (2019). A decomposition scheme for parallelization of system optimal dynamic traffic assignment on urban networks with multiple origins and destinations. Computer-Aided Civil and Infrastructure Engineering, 34(10), 915-931.

Nguyen, S., \& Dupuis, C. (1984). An efficient method for computing traffic equilibria in networks with asymmetric transportation costs. Transportation Science, 18(2), 185202.

Nogal, M., \& Honfi, D. (2019). Assessment of road traffic resilience assuming stochastic user behaviour. Reliability Engineering \& System Safety, 185, 72-83. 
Nogal, M., O'Connor, A., Caulfield, B., \& Martinez-Pastor, B. (2016). Resilience of traffic networks: From perturbation to recovery via a dynamic restricted equilibrium model. Reliability Engineering \& System Safety, 156, 84-96.

Ouyang, M., \& Fang, Y. (2017). A mathematical framework to optimize critical infrastructure resilience against intentional attacks. Computer-Aided Civil and Infrastructure Engineering, 32(11), 909-929.

Roy, K. C., Hasan, S., \& Mozumder, P. (2020). A multilabel classification approach to identify hurricaneinduced infrastructure disruptions using social media data. Computer-Aided Civil and Infrastructure Engineering, 35(12), 1387-1402.

Sharma, N., Tabandeh, A., \& Gardoni, P. (2020). Regional resilience analysis: A multiscale approach to optimize the resilience of interdependent infrastructure. ComputerAided Civil and Infrastructure Engineering, 35(12), 13151330.

Szeto, W., \& Lo, H. K. (2006). Dynamic traffic assignment: properties and extensions. Transportmetrica, 2(1), 31-52.

Transport \& Environment. (2020, Jan.). Recharge EU: How many charge points will EU countries need by 2030 (Tech. Rep.). Transport \& Environment.

Ukkusuri, S. V., Han, L., \& Doan, K. (2012). Dynamic user equilibrium with a path based cell transmission model for general traffic networks. Transportation Research Part B: Methodological, 46(10), 1657-1684.

U.S. Department of Energy. (2019). Vehicle charging. https://www.energy.gov/eere/ electricvehicles/vehicle-charging. Accessed Feb. 28, 2020.

U.S. Department of Energy. (2020). FOTW \#1064, january 14, 2019: Median all-electric vehicle range grew from 73 miles in model year 2011 to 125 miles in model year 2018. https://www.energy.gov/eere/vehicles/ articles/fotw-1064-january $\backslash-14-2019$-median -all-electric-vehicle-range-grew-73-miles.

Accessed Apr. 28, 2020.

Venkatraman, P., \& Levin, M. W. (2019). A congestion-aware tabu search heuristic to solve the shared autonomous vehicle routing problem. Journal of Intelligent Transportation Systems, 1-13.

Wang, B., Dehghanian, P., Wang, S., \& Mitolo, M. (2019). Electrical safety considerations in large-scale electric vehicle charging stations. IEEE Transactions on Industry Applications, 55(6), 6603-6612.

Wang, H., Abdin, A. F., Fang, Y.-P., \& Zio, E. (2021). Supplementary material. https://github.com/lucky105/ Sioux-Falls-network-in-cell-representation.

Accessed April. 15, 2021.

Wang, H., Fang, Y.-P., \& Zio, E. (2021). Risk assessment of an electrical power system considering the influence of traffic congestion on a hypothetical scenario of electrified transportation system in New York state. IEEE Transactions on Intelligent Transportation Systems, 22(1), 142-155.

Wang, J., Kong, Y., \& Fu, T. (2019). Expressway crash risk prediction using back propagation neural network: A brief investigation on safety resilience. Accident Analysis \& Prevention, 124, 180-192.

Xie, S., Hu, Z., Wang, J., \& Chen, Y. (2020). The optimal planning of smart multi-energy systems incorporating transportation, natural gas and active distribution networks. Applied Energy, 269, 115006.

Zhang, C., Yao, W., Yang, Y., Huang, R., \& Mostafavi, A. (2020). Semiautomated social media analytics for sensing societal impacts due to community disruptions during disasters. Computer-Aided Civil and Infrastructure Engineering, 35(12), 1331-1348.

Zhang, X., \& Mahadevan, S. (2017). A bio-inspired approach to traffic network equilibrium assignment problem. IEEE transactions on cybernetics, 48(4), 1304-1315.

Zhang, X., Mahadevan, S., \& Goebel, K. (2019). Network reconfiguration for increasing transportation system resilience under extreme events. Risk analysis, 39(9), 2054-2075.

Zhang, X., Mahadevan, S., Sankararaman, S., \& Goebel, K. (2018). Resilience-based network design under uncertainty. Reliability Engineering \& System Safety, 169, 364-379.

Zhou, Y., Wang, J., \& Yang, H. (2019). Resilience of transportation systems: concepts and comprehensive review. IEEE Transactions on Intelligent Transportation Systems, 20(12), 4262-4276.

Zhu, F., \& Ukkusuri, S. V. (2013). A cell based dynamic system optimum model with non-holding back flows. Transportation Research Part C: Emerging Technologies, 36, 367-380.

Zhu, F., \& Ukkusuri, S. V. (2018). Modeling the proactive driving behavior of connected vehicles: A cell-based simulation approach. Computer-Aided Civil and Infrastructure Engineering, 33(4), 262-281.

Ziliaskopoulos, A. K. (2000). A linear programming model for the single destination system optimum dynamic traffic assignment problem. Transportation science, 34(1), 3749.

Zio, E., \& Aven, T. (2011). Uncertainties in smart grids behavior and modeling: What are the risks and vulnerabilities? how to analyze them? Energy Policy, 39(10), 6308-6320. 